\title{
Impact of intramuscular administration of lipid-soluble and water-soluble vehicles into regenerating muscle at the distinct phases of skeletal muscle regeneration
}

\author{
Ratchakrit Srikuea $^{1}\left[\right.$ Kanokwan Suhatcho $^{1}$
}

Received: 31 May 2017 / Accepted: 28 October 2017 / Published online: 13 November 2017

(C) The Physiological Society of Japan and Springer Japan KK 2017

\begin{abstract}
Interpretation on the effectiveness of potential substances to enhance skeletal muscle regeneration is difficult if an inappropriate vehicle is administered, since vehicle administration can directly enhance or suppress regenerative capacity. In the current study, intramuscular administration of lipid-soluble and water-soluble vehicles into regenerating muscle at the distinct phases of skeletal muscle regeneration (regenerative vs. remodeling) were investigated. Tested vehicles included lipid-soluble [olive oil, $(0.1,1,5$, and $40 \%)$ dimethyl sulfoxide (DMSO), and $40 \%$ propylene glycol (PG)] and water-soluble $[0.9 \% \mathrm{NaCl}, \mathrm{PBS}, 0.1 \%$ ethanol, and distilled water]. Skeletal muscle regeneration was induced by $1.2 \% \mathrm{BaCl}_{2}$ injection to the tibialis anterior muscle of 10-week-old C57BL/6 male mice. Histological features, skeletal muscle stem cell activity, regenerating muscle fiber formation, angiogenesis, extracellular matrix remodeling, and macrophage infiltration were examined. The results revealed repeated administration of $40 \%$ DMSO and $40 \%$ PG causes significant recurrent muscle injury, which is pronounced during the remodeling phase compared to the regenerative phase. These findings were supported by (1) massive infiltration of $\mathrm{F} 4 / 80^{+}$macrophages; (2) significant increase of skeletal muscle stem cell re-activation and nascent regenerating muscle fiber formation; (3) excess fibrous formation; and (4) decreased regenerating muscle fiber cross-sectional area. These deleterious effects were comparable to $2 \%$ trypsin (degenerative substance) administration and less pronounced with a single administration. Nevertheless, recurrent muscle injury was still presented with 5\%
\end{abstract}

Ratchakrit Srikuea

ratchakrit.sri@mahidol.ac.th

1 Department of Physiology, Faculty of Science, Mahidol University, Bangkok 10400, Thailand
DMSO administration but it can be alleviated when $0.1 \%$ DMSO was administered during the remodeling phase. In contrast, none of the tested vehicles enhanced regenerative capacity compared with IGF-1 administration. Altogether, intramuscular administration of vehicle containing high concentration of DMSO or PG could impair skeletal muscle regenerative capacity and potentially affect validation of the investigational substance.

Keywords Dimethyl sulfoxide · Propylene glycol · Muscle injury $\cdot$ Regeneration $\cdot$ Remodeling $\cdot$ Satellite cell · Vehicle

\section{Introduction}

Skeletal muscle contains a well-characterized process to repair damaged muscle fibers after injury. This healing process requires the function of satellite cells, which are the resident skeletal muscle stem cells that become activated after muscle injury [1-4]. Skeletal muscle regeneration can be classified into two distinct phases: regenerative and remodeling. The regenerative phase involves a significant increase of myogenic regulatory factors (i.e., MyoD and myogenin) [5] and rapid differentiation of satellite cells that develop into nascent muscle fibers expressing embryonic myosin heavy chain (EbMHC) [6]. At this phase, angiogenesis is crucial and works to enhance regenerative muscle formation [7]. In contrast, the remodeling phase is a later event that coincides with the maturation of regenerating muscle fibers accompanied by extracellular matrix remodeling to organize the structural components. The significant up-regulation of extracellular matrix regulating gene transcriptional profiles after muscle injury support the importance of extracellular matrix remodeling during skeletal muscle regeneration [8]. 
Administrations of drugs [9-11], natural compounds $[12,13]$, growth factors [14-17], and potential substances [18-20] have been extensively investigated to test their beneficial effects on skeletal muscle regenerative capacity. However, administration of the investigational substance to the regenerating muscle requires the appropriate vehicle. Local administration, i.e., intramuscular administration, is beneficial, if prevention of the undesired effect of the investigational substance on non-target tissues is required. In addition, intramuscular administration enhances rate of delivery and rapid absorption of the testing substance [21] to the regenerating muscle compared to systemic administration routes, i.e., subcutaneous and intraperitoneal administrations.

Lipid-soluble and water-soluble vehicles for intramuscular administration are routinely used in biomedical research and clinical application. However, the direct effects of these vehicles on skeletal muscle regenerative capacity after intramuscular administration are currently unknown. Skeletal muscle regeneration is a complex process that involves satellite cell function, regenerative muscle formation, angiogenesis, extracellular matrix remodeling, and the up-regulation of various growth factors and cytokines all of which can influence the reparative process [1]. Therefore, it is likely that the vehicle itself could enhance or suppress the regenerative capacity through interaction with these regenerative components. Hence, the interpretation of the investigational substance can be influenced if an inappropriate vehicle is administered. Moreover, the administration frequency of the vehicle is directly related to the half-life of the investigational substance. Nevertheless, the effects of administration of vehicle at different frequencies during the distinct phases of skeletal muscle regeneration have not been explored. Therefore, the present study aims to delineate the effects of intramuscular administration of lipid-soluble and water-soluble vehicles on skeletal muscle regenerative capacity at the distinct phases of regeneration (regenerative vs. remodeling) with different administration frequencies (single injection vs. multiple injections).

\section{Materials and methods}

\section{Animals}

Male C57BL/6 mice were obtained from the National Laboratory Animal Centre, Salaya, Nakhon Pathom, Thailand. Mice were housed at the Laboratory Animal Facilities (Faculty of Science, Mahidol University) under 12:12-h light-dark cycle in a temperature- and humidity-controlled room. Experimental procedures in animals were conducted in accordance with institutional guidelines for the care and use of laboratory animals which was approved by the Faculty of Science, Mahidol University Animal Care and Use Committee (SCMU-ACUC) (Protocol No. MUSC57-014-309).

\section{Chemicals and reagents}

All chemicals and reagents were purchased as listed follows: olive oil (O1514), LONG ${ }^{\circledR} \mathrm{R}^{3}$ IGF-I human recombinant analog (I1271), 2-methylbutane (M32631), Triton ${ }^{\circledR}$ X-100 (X100), mouse monoclonal anti-dystrophin antibody (D8168), and rabbit polyclonal anti-laminin antibody (L9393) (Sigma, St Louis, MO, USA); mouse monoclonal anti-myogenin (sc-12732), anti-MYH3 (F1.652) (sc-53091), and anti-vimentin (sc-32322) antibodies (Santa Cruz Biotechnology, CA, USA); rabbit polyclonal anti-CD31 (ab28364), rat monoclonal anti-F4/80 (ab6640), and goat anti-rat Alexa Fluor ${ }^{\circledR} 488$ (ab150157) antibodies (Abcam, Cambridge, UK); dimethyl sulfoxide (DMSO) (102952), ethanol (EtOH) (108543), acetone (100014), hematoxylin solution modified acc. to Gill II (105175), eosin Y (C.I. 45380) (115935), and ImmunoPen ${ }^{\mathrm{TM}}$ (402176) (Merck Millipore, MA, USA); normal goat serum (PCN5000), goat anti-mouse Alexa Fluor ${ }^{\circledR} 568$ (A-11004), and goat anti-rabbit Alexa Fluor $^{\circledR} 488$ secondary antibodies (A-11008) (Invitrogen, CA, USA); mouse IgG blocking reagent (MKB-2213) and antifade containing DAPI mounting medium (H-1200) (Vector Laboratories, CA, USA); Tissue-Tek O.C.T. Compound (4583) (Sakura Finetek, CA, USA); Xylene (X/0250/17) and Permount ${ }^{\mathrm{TM}}$ Mounting Medium (SP15) (Fisher Scientific, NJ, USA); paraformaldehyde (PFA) (15713) (Electron Microscopy Science, PA, USA); sterile phosphate-buffered saline (PBS) (10010023) and trypsin solution, no phenol red (15090046) (Gibco, NY, USA); Attane ${ }^{\mathrm{TM}}$ Isoflurane, USP (Piramal Critical Care, Inc., PA, USA); barium chloride (AJA81) and propylene glycol (PG) (AJA427) (Ajax Finechem Pty Ltd, NSW, Australia).

\section{Skeletal muscle regeneration study model}

Young adult male mice (10-week-old) were anesthetized with isoflurane prior intramuscular administration of $50 \mu \mathrm{l}$ of $1.2 \% \mathrm{BaCl}_{2}$ solution to the tibialis anterior (TA) muscle. During injection, $1.2 \% \mathrm{BaCl}_{2}$ solution was slowly released into the TA muscle to induce extensive injury and skeletal muscle regeneration. Thereafter, mice were assigned into two groups to investigate the phases of skeletal muscle regeneration. Characteristics of regenerative and remodeling phases were determined according to the expression levels of EbMHC and myogenin (myogenic regulatory factor) proteins. Skeletal muscle cross-sectional area (CSA) and fiber size distribution were examined to verify the changes of histological perspective at these distinct phases of skeletal muscle regeneration. 


\section{Intramuscular administration of vehicles}

Mice were randomly allocated into four groups to investigate the effects of intramuscular administration of lipid-soluble and water-soluble vehicles on skeletal muscle regenerative capacity. Regenerating TA muscles received intramuscular administration of $50 \mu \mathrm{l}$ of lipid-soluble vehicles [olive oil, $(0.1,1,5$, and $40 \%)$ DMSO, and $40 \%$ PG] or water-soluble vehicles $(0.9 \% \mathrm{NaCl}$, PBS, $0.1 \% \mathrm{EtOH}$, and distilled water) at the distinct phases of skeletal muscle regeneration with different administration frequencies including (1) single injection at regenerative phase (single administration on day 4 after $\mathrm{BaCl}_{2}$ injection); (2) multiple injections at regenerative phase (repeated administration once a day for 4 days during day 4-7 after $\mathrm{BaCl}_{2}$ injection); (3) single injection at remodeling phase (single administration on day 11 after $\mathrm{BaCl}_{2}$ injection); and (4) multiple injections at remodeling phase (repeated administration once a day for 4 days during day 11-14 after $\mathrm{BaCl}_{2}$ injection). DMSO $(0.1,1,5$, and $40 \%)$ and PG (40\%) were prepared in $0.9 \% \mathrm{NaCl}$ and distilled water, respectively. No vehicle administration (needle puncture) serves as a respective control in this study. Moreover, two substances that have been proven to act as a growth factor (insulin-like growth factor-1; IGF-1) and trypsin (degenerative substance) on skeletal muscle regenerative process were investigated in the current study to interpret the effects of different vehicles on skeletal muscle regenerative capacity. IGF-1 is a growth factor that has been reported to promote skeletal muscle regeneration after injury $[15,17$, 22] and its effective dose for intramuscular administration was $100 \mathrm{ng} /$ injection [23]. In contrast to IGF-1, 2\% trypsin can induce an impairment on skeletal muscle regeneration process by its destructive effect on the basal lamina of skeletal muscle fibers [24]. The administrations of IGF-1 and $2 \%$ trypsin were performed according to the experimental design as described for vehicle administrations.

\section{Muscle sample collection}

Regenerating TA muscles were collected on 8 days (regenerative phase) and 15 days (remodeling phase) after $\mathrm{BaCl}_{2}$-induced muscle injury, TA muscle was finely dissected and muscle wet weight was determined using a digital weight scale (Mettler Toledo, MS204S). Normalization of TA muscle wet weight (mg) to mouse body weight $(\mathrm{g})$ represents the muscle wet weight to body weight ratio.

\section{Histological analysis}

Muscle samples were placed in O.C.T. compound, snap frozen in pre-cooled 2-methylbutane, and sectioned at $10 \mu \mathrm{m}$ thickness with cryostat (CM1850; Leica, Wetzlar, Germany). Histological features were determined using hematoxylin and eosin staining for an evaluation of regenerating muscle fiber morphology. For regenerating muscle fiber CSA quantification, six images were randomly captured through the entire regenerating tibialis anterior section at $\times 200$ magnification using Olympus microscope (Model BX53) connected with a digital camera (DP73) (Olympus, Tokyo, Japan). Regenerating muscle fiber CSA was analyzed using ImageJ 1.50i (National Institutes of Health, Bethesda, MD, USA).

\section{Immunohistochemical analysis}

All immunostaining steps for EbMHC (encoded by the MYH3 gene), myogenin, CD31, and vimentin were performed as previously described [25]. Laminin and dystrophin antibodies were applied for double-labeling study to delineate regenerating muscle fiber structure. For F4/80 and dystrophin double-labeling, sections were pre-fixed with icecold acetone for $10 \mathrm{~min}$. After washed with PBS, the sections were blocked with mouse IgG blocking reagent for $1 \mathrm{~h}$ to reduce non-specific staining that could develop when apply the primary antibody raised from mouse on mouse tissue. Thereafter, sections were washed with PBS, $10 \%$ normal goat serum was applied for $1 \mathrm{~h}$ followed with simultaneous incubation of primary rat anti-F4/80 antibody (1:100) and mouse anti-dystrophin antibody (1:100) overnight at $4{ }^{\circ} \mathrm{C}$. For immunofluorescence signal visualization, the stainedsections were washed with PBS followed with simultaneous incubation of goat anti-rat Alexa Fluor ${ }^{\circledR} 488$ (1:500) and goat anti-mouse Alexa Fluor ${ }^{\circledR} 568$ (1:500) secondary antibodies for $1 \mathrm{~h}$ in the dark. Thereafter, the stained-sections were extensively washed with PBS $+0.01 \%$ Tween ${ }^{\circledR} 20$ to washout excess secondary antibodies. Anti-fade containing DAPI was applied for nuclear visualization and preserving the fluorescent-stained signal. For quantitative analysis, the mid-belly portion of regenerating TA muscle was used to analyze. Six images were obtained from randomly captured through the entire regenerating tibialis anterior section using Olympus Microscope (Model BX53) connected with a digital camera (DP73) (Olympus) at $\times 200$ magnification. The quantitative area of expressions of EbMHC, CD31, and vimentin proteins, and $\mathrm{F} 4 / 80^{+}$cell infiltration were analyzed using segmentation method while myogenin ${ }^{+}$nuclei were counted using single point measurement tool of cellSens Dimension Desktop software version 1.14 (Olympus). EbMHC, CD31, and vimentin area of expressions, F4/80 ${ }^{+}$ cell infiltration, and number of myogenin ${ }^{+}$nuclei from vehicle, IGF-1, and $2 \%$ trypsin administered groups were normalized with the data of no vehicle group and expressed as fold change for quantitative analysis. The representative immunostaining images were illustrated with either $\times 200$ or $\times 400$ magnification. 


\section{Statistical analysis}

Data are presented as mean \pm SEM. Normal distribution and the homogeneity of variance were analyzed with Shapiro-Wilk and Levene's test, respectively. Independent $t$ test, Mann-Whitney $U$ test, one-way ANOVA with Dunnett or Dunnett's T3 post hoc test were used to compare the differences between groups. The significant differences among groups were analyzed with SPSS version 18.0 and the statistical significant was set at $p<0.05$.

\section{Results}

Characteristics of regenerative and remodeling phases of skeletal muscle regeneration

Regenerative and remodeling phases of skeletal muscle regeneration are distinct processes related to EbMHC protein expression and satellite cell activity. Regenerative phase is classified as the stage that robust EbMHC protein expression accompanies the rapid differentiation of satellite cells (myogenin ${ }^{+}$nuclei, arrowheads) (Fig. 1a, b, left panel). On

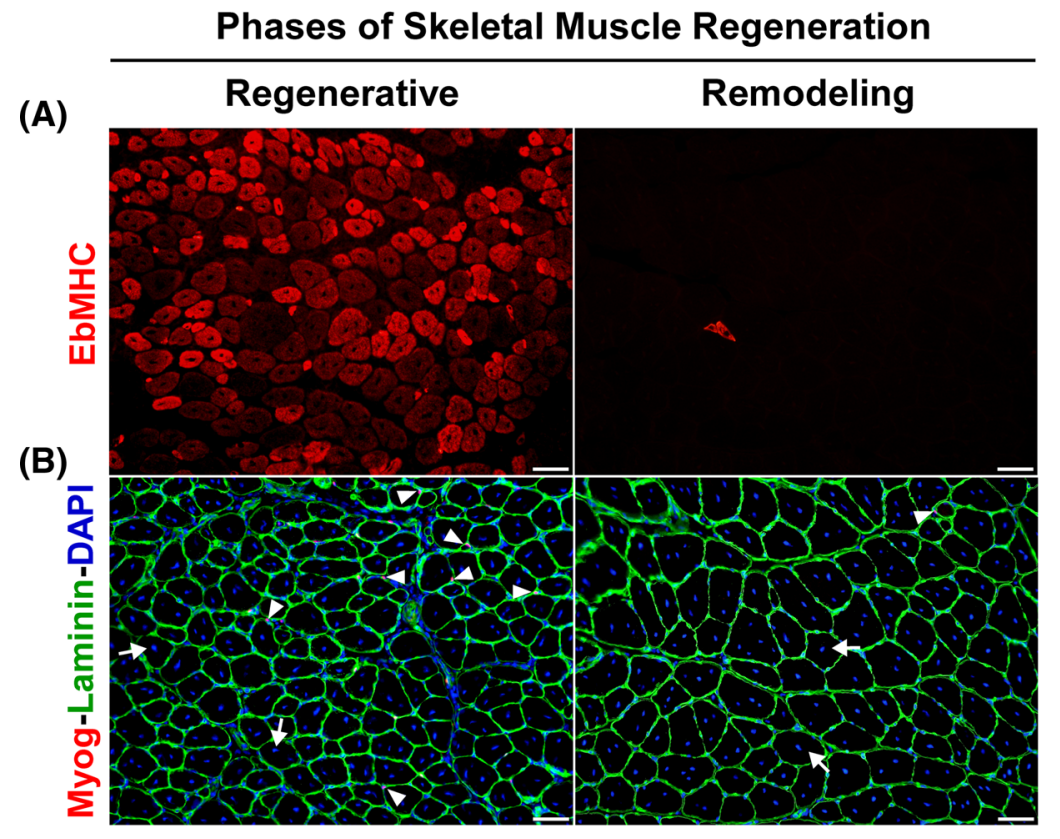

(C)

(D)

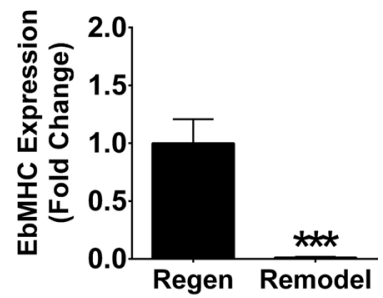

(E)

(F)
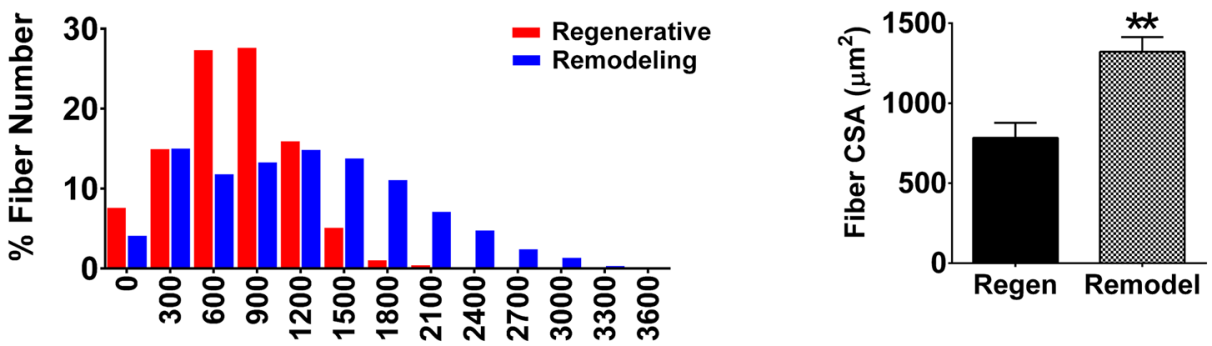

Fiber Cross-Sectional Area $\left(\mu \mathrm{m}^{2}\right)$

Fig. 1 Characteristics of regenerative and remodeling phases of skeletal muscle regeneration. a Representative images of EbMHC immunostaining and $\mathbf{b}$ myogenin ${ }^{+}$nuclei (arrowheads) during regenerative and remodeling phases, scale bars $=50 \mu \mathrm{m}$. Laminin and DAPI were applied to delineate regenerating muscle fiber structure and nuclei, respectively. Arrows indicate centrally nucleated muscle fibers (hallmark of skeletal muscle regeneration). c, d Quantitative analysis of EbMHC and myogenin protein expression. $* * * p<0.001$ compared to regenerative phase ( $n=5$ mice/group). e Histogram analysis of regenerating muscle fiber CSA during regenerative and remodeling phases. The average number of measured regenerating muscle fiber CSA during regenerative and remodeling phases were 1240 and 797 fibers/mice ( $n=5$ mice/group). $\mathbf{f}$ Quantitative analysis of regenerating fiber CSA during regenerative and remodeling phases, $* * p<0.01$ compared to regenerative phase ( $n=5$ mice/group). Regen regenerative phase, Remodel remodeling phase 
the other hand, the remodeling phase is characterized as the stage that barely detectable of EbMHC protein expression and low satellite cell differentiation (Fig. 1a, b, right panel). This remodeling phase represents the maturation of regenerating muscle fibers and extracellular matrix remodeling. To verify these distinct processes of skeletal muscle regeneration, significantly lower of EbMHC protein expression $(0.01 \pm 0.01$-fold $)(p<0.001)$ (Fig. 1c) and myogenin protein expression $(0.09 \pm 0.03$-fold $)(p<0.001)$ (Fig. 1d) in the remodeling phase compared to regenerative phase is apparent. In histological perspective, the sharing characteristic between these two phases of skeletal muscle regeneration is the presence of centrally nucleated regenerating muscle fibers as illustrated in Fig. 1b (arrows). Nevertheless, the difference in regenerating muscle fiber CSA between the regenerative and remodeling phases was evident. As histogram analysis revealed a significant increase in regenerating muscle fiber size (rightward shift) in the remodeling phase compared to the regenerative phase (Fig. 1e) that associated with a significant increase of regenerating fiber CSA $\left(784 \pm 41.7\right.$ vs. $\left.1321.0 \pm 92.4 \mu^{2}\right)(p<0.01)$ (Fig. 1f).

\section{Impact of intramuscular administration of vehicles during the regenerative phase}

To investigate the effects of intramuscular administration of vehicles during the regenerative phase, regenerating TA muscles received daily intramuscular administration of vehicle compounds during days $4-7$ after $1.2 \% \mathrm{BaCl}_{2}$ injection. Four to seven days after injury induction represents the regenerative phase closely related to the substantial expression of skeletal muscle regeneration regulatory proteins (EbMHC and myogenin) and angiogenesis. This phase occurs following the degenerative phase that involves an extensive degeneration of the damaged muscle fibers. In this experiment, histological analysis demonstrated an increase in small nascent regenerating muscle fibers in $40 \%$ DMSO, $40 \%$ PG, and $2 \%$ trypsin administered groups compared to no vehicle (Fig. 2a). These changes were correlated with the tendency to increase in EbMHC and myogenin protein expression in $40 \%$ DMSO (1.4 \pm 0.1 -fold and $1.7 \pm 0.3$-fold $)$ and $40 \%$ PG (1.5 \pm 0.2 -fold and $1.5 \pm 0.2$-fold) administered groups (Fig. 2b, c). Moreover, intramuscular administration of these vehicles tended to alter CD31 protein expression $(0.8 \pm 0.1$-fold and $0.9 \pm 0.1$-fold) (Fig. $2 \mathrm{~d}$ ) but failed to change muscle wet weight to body weight ratio compared to no vehicle (Fig. 2e). On the other hand, EbMHC protein expression was decreased after IGF-1 administration compared to no vehicle group $(0.4 \pm 0.1$-fold $)(p<0.05)$ (Fig. 2b). Furthermore, there was a significant increase in EbMHC (1.6 \pm 0.2 -fold) and a decrease in CD31 (0.8 \pm 0.1 fold) protein expression in $2 \%$ trypsin administered group compared to no vehicle $(p<0.05)$ (Fig. $2 \mathrm{~b}, \mathrm{~d})$. However, daily intramuscular administrations of olive oil, $0.9 \% \mathrm{NaCl}$, PBS, $0.1 \% \mathrm{EtOH}$, and distilled water at the regenerative phase did not impact skeletal muscle regulatory protein expression (Fig. 2b, c), angiogenesis (Fig. 2d), or muscle wet weight to body weight ratio (Fig. 2e).

\section{Immunohistochemical perspectives of intramuscular administrations of $40 \%$ DMSO, $40 \%$ PG, IGF-1, and $2 \%$ trypsin during the regenerative phase}

The representative images of EbMHC, myogenin, and CD31 protein expression after daily intramuscular administrations of $40 \%$ DMSO, $40 \%$ PG, IGF-1, and $2 \%$ trypsin compared to no vehicle during the regenerative phase are illustrated in Fig. 3a. The small nascent regenerating muscle fibers with robust EbMHC protein expression (arrows) were apparent in $40 \%$ DMSO, $40 \%$ PG, and 2\% trypsin administered groups. These changes were related with a tendency to increase myogenin (arrowheads) and decrease CD31 protein expression (Fig. 3a). In contrast, barely detection of EbMHC and decreased CD31 protein expression was evident in IGF-1 and $2 \%$ trypsin administered groups, respectively. Representative double-labeling images of EbMHC, myogenin, and CD31 protein expression with laminin/dystrophin at the defective area that comparably observed in $40 \%$ DMSO, $40 \%$ PG, and $2 \%$ trypsin administered groups are illustrated in Fig. $3 b$.

\section{Impact of intramuscular administration of vehicles during the remodeling phase}

In contrast to a rapid repair of damaged muscle fibers during the regenerative phase, the remodeling phase accounts for the enlargement of the regenerating muscle fibers and extracellular matrix remodeling to re-organize the structural components. In this subsequent experiment, regenerating TA muscles received daily intramuscular administration of vehicle compounds for 4 consecutive days during days 11-14 after muscle injury. This administration schedule encompasses the remodeling phase of skeletal muscle regeneration. After multiple intramuscular administrations of vehicle compounds were applied at this phase, massive increased of nascent regenerating muscle fibers in 40\% DMSO, $40 \%$ PG, and 2\% trypsin administered groups was evident (Fig. 4a). These results suggest intramuscular administration of $40 \%$ DMSO, $40 \%$ PG, and 2\% trypsin leads to a significant recurrent muscle injury. To support this notion, skeletal muscle regeneration regulatory proteins (EbMHC and myogenin) were significantly increased in 40\% DMSO [27.0 \pm 2.8 -fold and $8.5 \pm 1.2$-fold $(p<0.01)], 40 \%$ PG [33.3 \pm 5.2 -fold and $6.4 \pm 0.9$-fold $(p<0.01)]$, and $2 \%$ trypsin [53.5 \pm 3.6 -fold and $7.4 \pm 1.4$-fold $(p<0.01)]$ administered groups compared to no vehicle (Fig. 4b, c). Moreover, muscular fibrosis 


\section{Regenerative Phase of Skeletal Muscle Regeneration}

(A)
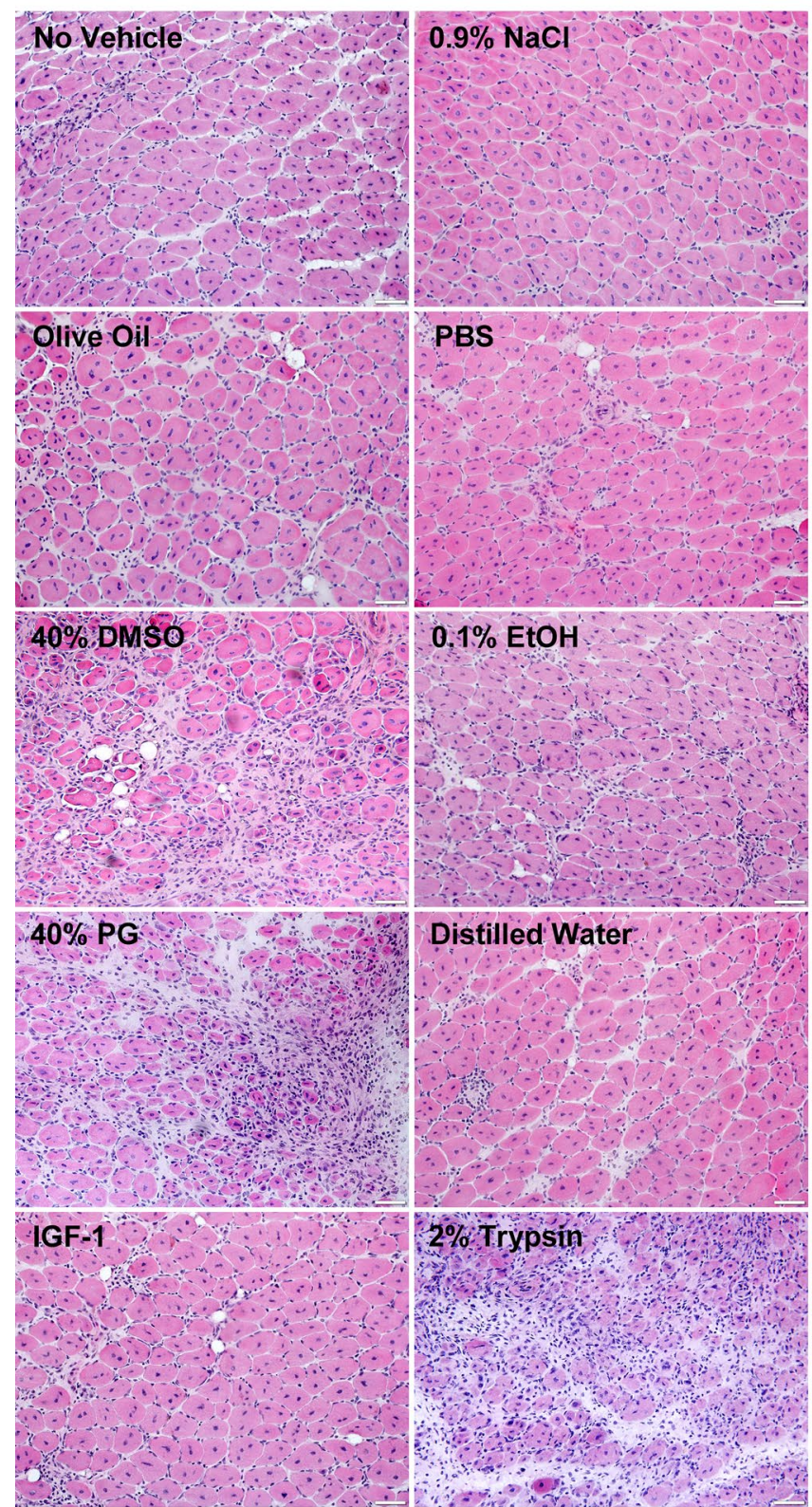

(B)

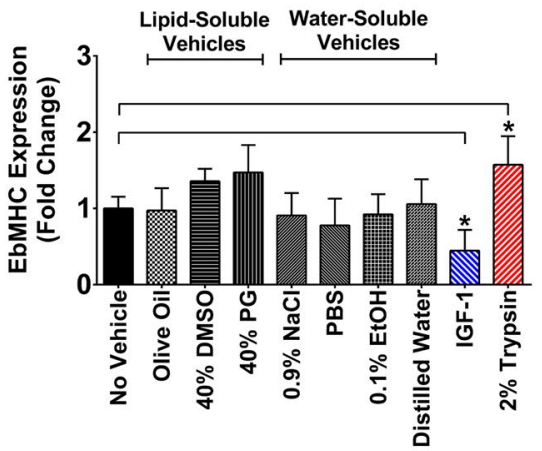

(C)

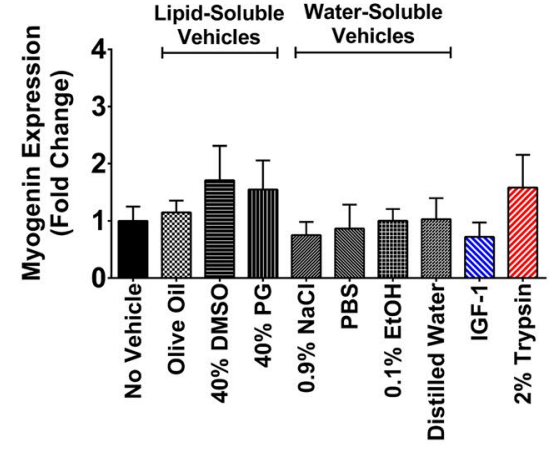

(D)

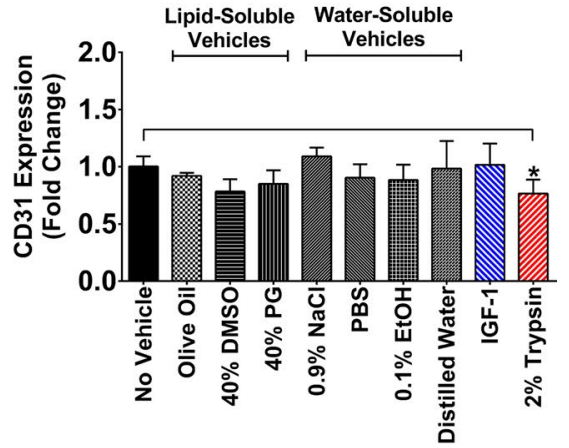

(E)

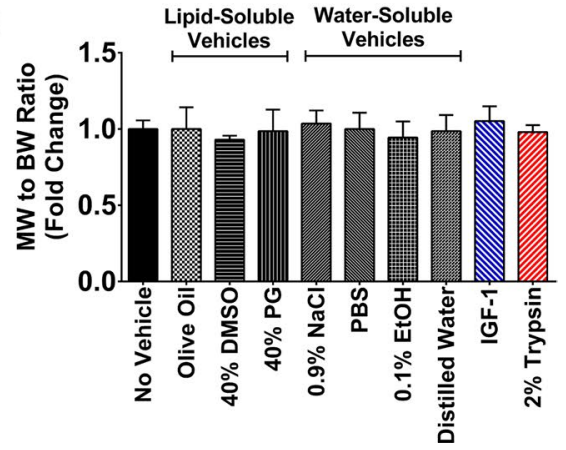

Fig. 2 Impact of intramuscular administration of lipid-soluble or water-soluble vehicles during the regenerative phase of skeletal muscle regeneration. a $\mathrm{H} \& \mathrm{E}$ staining illustrates histological features of regenerating TA muscle following intramuscular administration of vehicle compounds. Muscle samples were collected on day
8 post-injury. Images were captured at $\times 200$ magnification, scale bars $=50 \mu \mathrm{m}$. b-d Quantitative analysis of EbMHC, myogenin, and CD31 protein expression and e muscle wet weight to body weight ratio. $* p<0.05$ compared to no vehicle ( $n=5$ mice/group) 
Fig. 3 Impact of intramuscular administrations of $40 \%$ DMSO, $40 \%$ PG, IGF- 1 , and $2 \%$ trypsin on skeletal muscle regenerative capacity during the regenerative phase. a Representative images of skeletal muscle regeneration regulatory proteins (EbMHC and myogenin) and CD31 protein expression compared to no vehicle. Images were captured at $\times 200$ magnification, scale bars $=50 \mu \mathrm{m}$. b Representative images of EbMHC, myogenin, and CD31 protein expression in the defective areas at $\times 400$ magnification (serial sections are denoted by asterisks), scale bars $=50 \mu \mathrm{m}$. Arrows and arrowheads indicate the localization of nascent regenerating muscle fibers with robust EbMHC and myogenin protein expression, respectively. Laminin and dystrophin were applied to delineate regenerating muscle fiber structure and nuclei were visualized with DAPI

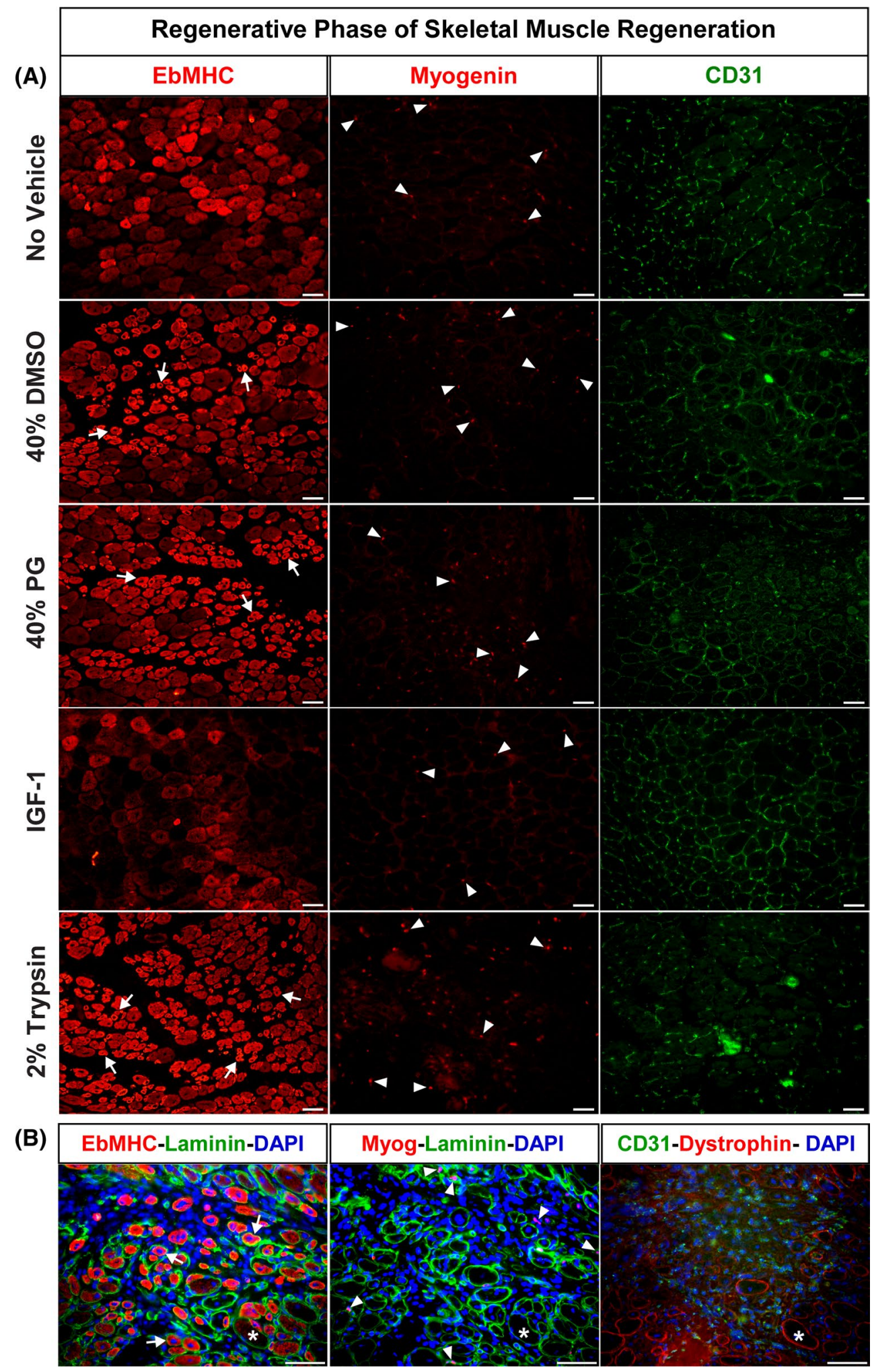

associated with increased vimentin protein expression was evident in $40 \%$ DMSO (2.2 \pm 0.2 -fold), $40 \%$ PG $(2.1 \pm 0.1$ fold), and $2 \%$ trypsin $(2.6 \pm 0.3$-fold $)$ administered groups $(p<0.001)$ (Fig. 4d). In conjunction with these changes, there was a significant reduction in muscle wet weight to body weight ratio following administration of $40 \%$ DMSO $(0.87 \pm 0.03$-fold, $p<0.05), 40 \%$ PG $(0.87 \pm 0.03$-fold, $p<0.05)$, and $2 \%$ trypsin $(0.85 \pm 0.03$-fold, $p<0.01)$ 
(Fig. 4e). Nevertheless, no significant differences in histological features, skeletal muscle regeneration regulatory protein expression, fibrous formation, and muscle wet weight to body weight ratio in $0.9 \% \mathrm{NaCl}, \mathrm{PBS}$, and distilled water administered groups existed when compared to no vehicle (Fig. 4a-e). In contrast, multiple administrations of $0.1 \%$ EtOH at the remodeling phase induced a small degenerative area (Fig. 4a) that was paralleled with a tendency to increase EbMHC protein expression $(6.3 \pm 1.9$-fold $)$ and a significant increase of myogenin protein expression $(2.6 \pm 0.4$-fold $)$ $(p<0.05)$ (Fig. 4b, c). Moreover, excess oil accumulation in the subcutaneous (extramuscular space) after repeated administration of olive oil was observed. This condition lead to the tendency to reduce muscle wet weight to body weight ratio $(0.93 \pm 0.02$-fold $)$ compared to no vehicle group (Fig. 4e).

\section{Immunohistochemical perspectives of intramuscular administrations of $40 \%$ DMSO, $40 \%$ PG, IGF-1, and $2 \%$ trypsin during the remodeling phase}

The representative images of $40 \%$ DMSO, $40 \%$ PG, IGF-1, and $2 \%$ trypsin administered groups during the remodeling phase compared to no vehicle are illustrated in Fig. 5a. In contrast to the regenerative phase, EbMHC and myogenin protein expression were barely detected in the no vehicle group. However, a significant increase in EbMHC protein expression in the nascent regenerating muscle fibers (arrows) and myogenin protein expression (arrowheads) associated with increased vimentin protein expression were evident in 40\% DMSO, 40\% PG, and 2\% trypsin administered groups (Fig. 5a). Representative double-labeling images of EbMHC, myogenin, and vimentin protein expression with laminin at the defective area that comparably observed in $40 \%$ DMSO, $40 \% \mathrm{PG}$, and $2 \%$ trypsin administered groups are illustrated Fig. 5b. In contrast, IGF-1 administration had no significant impact on regenerating muscle fiber maturation at the remodeling phase as indicated by no significant differences of EbMHC and myogenin protein expression compared to no vehicle group (Fig. 5a).

\section{Effects of administration frequency of vehicles at the regenerative and remodeling phases}

Administration frequency is determined by the half-life of the testing substance; however, the effect of intramuscular administration of vehicle compounds with different frequencies at the distinct phases of skeletal muscle regeneration on regenerative capacity is not yet known. Hence, comparison of the effects of single administration and multiple administrations of vehicle compounds on skeletal muscle regenerative capacity at regenerative and remodeling phases were verified. In this analysis, the single administration of vehicle was performed at the initial period of regenerative phase or remodeling phase, respectively. The results revealed significant impact of administration frequency in $40 \%$ DMSO, $40 \%$ PG, and $2 \%$ trypsin administered groups compared to no vehicle at the remodeling but not regenerative phase. Multiple administrations of $40 \%$ DMSO, $40 \%$ PG, and $2 \%$ trypsin at the remodeling phase lead to a significant increase of EbMHC protein expression 2.3- and 2.6-fold ( $p<0.01$ ), and 5.4-fold ( $p<0.001)$, myogenin protein expression (3.4-, 2.7 -, and 6.4-fold) ( $p<0.01)$, and vimentin protein expression (1.5-, 1.3-, and 1.9-fold) $(p<0.05)$ compared to single administration.

\section{Recurrent muscle injury after intramuscular administrations of $40 \%$ DMSO, $40 \%$ PG, and $2 \%$ trypsin}

Previous experiments showed recurring muscle injury and impeded skeletal muscle regeneration after repeated administration of $40 \%$ DMSO, $40 \% \mathrm{PG}$, and $2 \%$ trypsin during the remodeling phase. To confirm these observations, the abundance of macrophage infiltration that associated with the presence of nascent regenerating muscle fiber was analyzed (Fig. 6a). F4/80 ${ }^{+}$macrophage infiltration (arrowheads) was explicitly seen in 40\% DMSO, $40 \%$ PG, and $2 \%$ trypsin administered groups compared to no vehicle (Fig. 6b). Quantitative analysis confirmed a significant increase in $\mathrm{F} 4 / 80^{+}$infiltrated cells in $40 \%$ DMSO (21.7 \pm 5.0 -fold), 40\% PG (19.0 \pm 5.5-fold), and $2 \%$ trypsin (29.2 \pm 4.1 -fold) administered groups compared to no vehicle group ( $p<0.001)$ (Fig. 6c). These changes were paralleled with a decrease in regenerating muscle fiber CSA in 40\% DMSO $\left(777.8 \pm 47.7 \mu \mathrm{m}^{2}\right), 40 \%$ PG $\left(988.7 \pm 86.4 \mu \mathrm{m}^{2}\right)$, and $2 \%$ trypsin $\left(921.2 \pm 72.7 \mu \mathrm{m}^{2}\right)$ administered groups compared to no vehicle group $\left(1371.0 \pm 77.1 \mu \mathrm{m}^{2}\right)(p<0.01)$. To support this notion, a leftward shift of regenerating muscle fiber CSA in $40 \%$ DMSO, 40\% PG, and 2\% trypsin administered groups was evident as illustrated in Fig. 6d. Altogether, these results provide solid evidence supporting recurrent muscle injury after repeated intramuscular administration of $40 \%$ DMSO, $40 \%$ PG, and 2\% trypsin during the remodeling phase of skeletal muscle regeneration. In contrast, no 


\section{Remodeling Phase of Skeletal Muscle Regeneration}

(A)
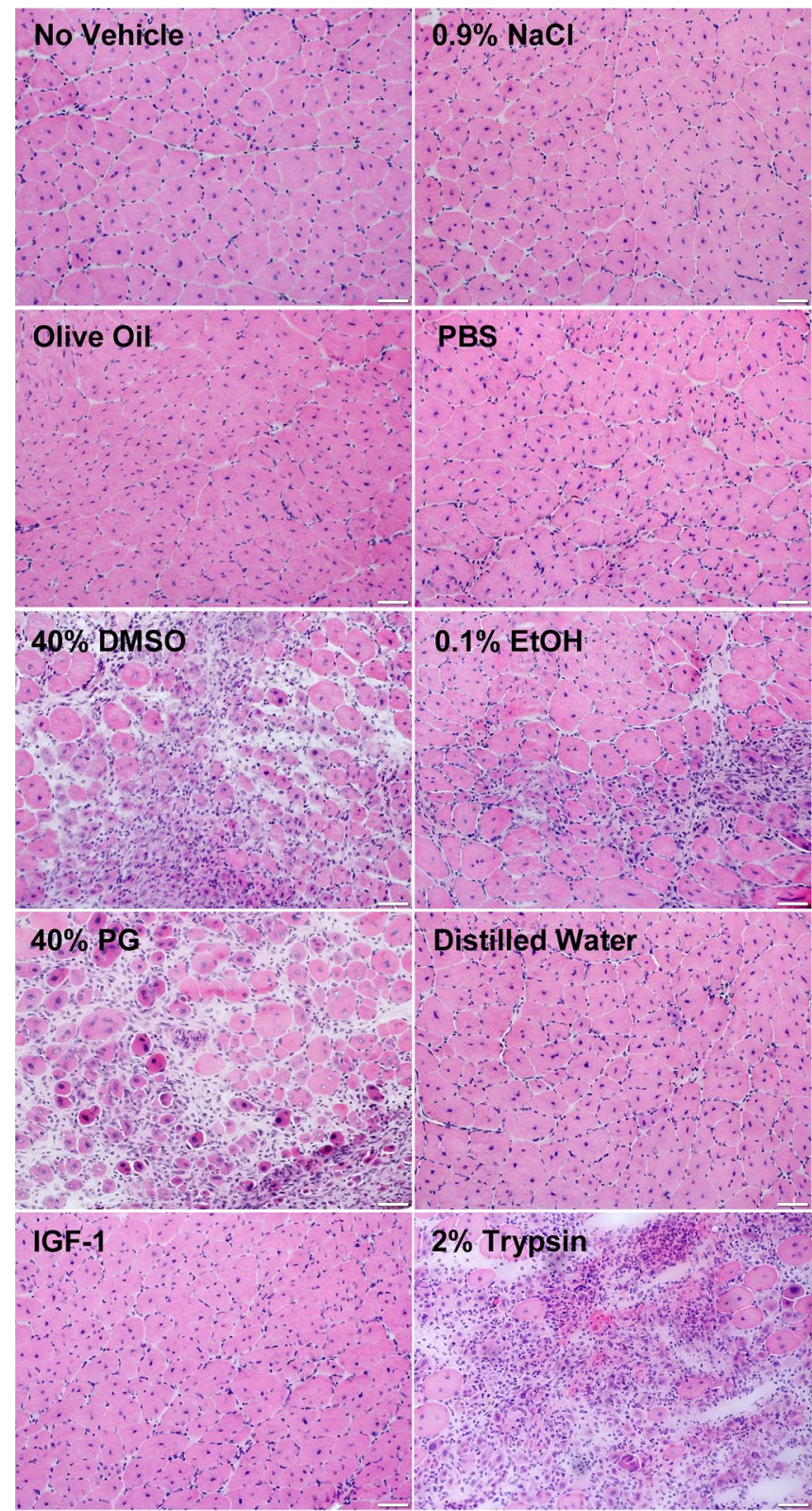

(B)

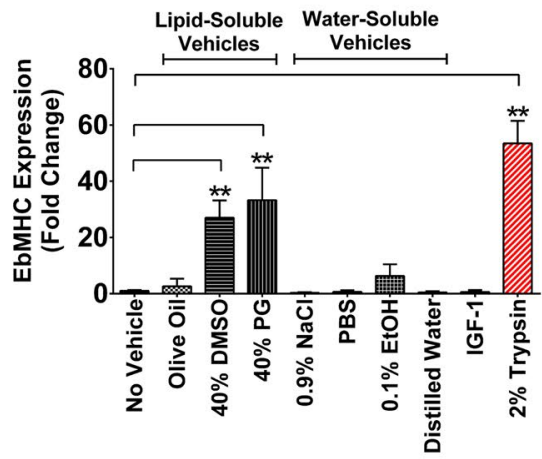

(C)

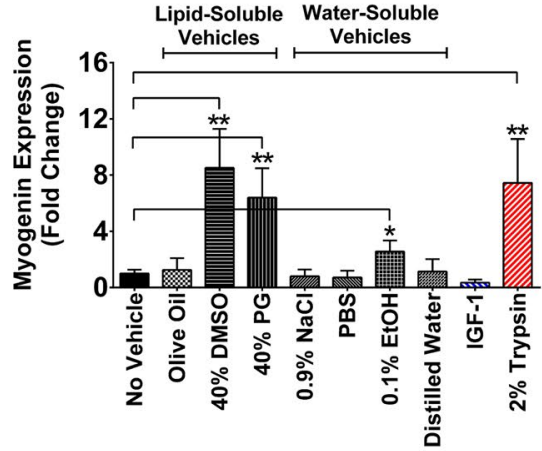

(D)

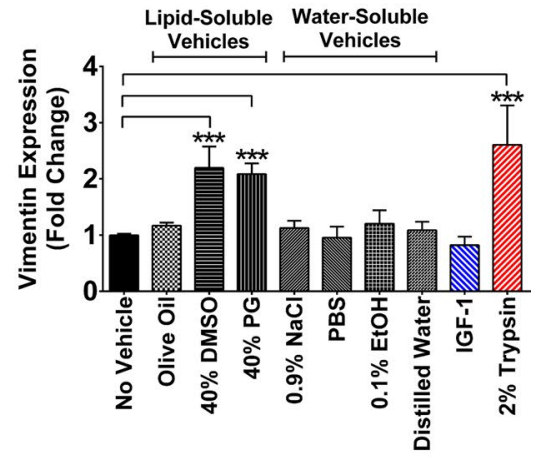

(E)

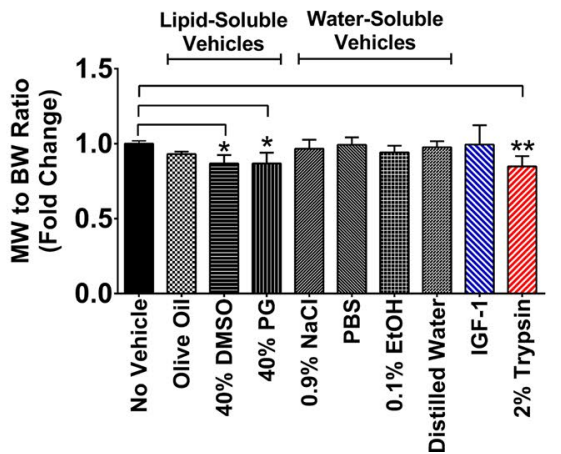

Fig. 4 Impact of intramuscular administration of lipid-soluble or water-soluble vehicles during the remodeling phase of skeletal muscle regeneration. a H\&E staining illustrated histological features of regenerating TA muscle after intramuscular administration of vehicle compounds. Muscle samples were collected on day 15 post-injury.
Images were captured at $\times 200$ magnification, scale bars $=50 \mu \mathrm{m}$. bd Quantitative analysis of EbMHC, myogenin, and vimentin protein expression and e muscle wet weight to body weight ratio. $* p<0.05$, $* * p<0.01$, and $* * * p<0.001$ compared to no vehicle $(n=5 \mathrm{mice} /$ group) 
significant difference of $\mathrm{F} 4 / 80^{+}$infiltrated cells in olive oil, $\mathrm{NaCl}$, PBS, distilled water, and IGF-1 administered groups compared to no vehicle group, however, tend to increase $\mathrm{F} 4 / 80^{+}$infiltrated cells in $0.1 \% \mathrm{EtOH}$ administered group was demonstrated (Fig. 6c).

\section{Impact of intramuscular administration of low concentrations of DMSO during the remodeling phase}

Since DMSO is widely used as a vehicle for skeletal muscle regeneration study, however, intramuscular administration using high concentration of DMSO (40\%) is highly toxic to the regenerating muscle during the remodeling phase. Therefore, the safety concentration of DMSO that has no deleterious effect to the regenerating muscle was further investigated. In this experiment, low concentrations of DMSO $(0.1$, 1 , and $5 \%$ ) were intramuscular administered to the regenerating muscle during the remodeling phase of skeletal muscle regeneration. The results revealed that $0.1 \%$ DMSO administration had no significant effects on EbMHC (Fig. 7a), myogenin (Fig. 7b), and vimentin (Fig. 7c) protein expression, F4/80 $0^{+}$infiltrated cells (Fig. 7d), and regenerating muscle fiber CSA (Fig. 7e) compared to no vehicle group. When the concentration of DMSO was increased up to $1 \%$, no significant impact on EbMHC, myogenin, and vimentin protein expression (Fig. 7a-c) still observed but $\mathrm{F} 4 / 80^{+}$infiltrated cells was significantly increased $(5.6 \pm 1.1$-fold $)(p<0.05)$ (Fig. 7d) compared to no vehicle. Nevertheless, recurrent muscle injury was evident after 5\% DMSO was intramuscular administered. Significantly increased of EbMHC protein expression ( $8.4 \pm 1.5$-fold) $(p<0.01)$ (Fig. 7a), myogenin protein expression $(2.5 \pm 0.4$-fold $)(p<0.05)$ (Fig. $7 b)$, vimentin protein expression $(1.5 \pm 0.04$-fold $)(p<0.001)$ (Fig. 7c), and F4/80 ${ }^{+}$infiltrated cells $(11.3 \pm 1.9$-fold $)$ $(p<0.01)$ (Fig. 7d) were demonstrated. These changes were related to a significant decreased of regenerating muscle fiber CSA $\left(965.4 \pm 24.6 \mu \mathrm{m}^{2}\right)(p<0.01)$ (Fig. 7e) and a leftward shift of regenerating muscle fiber size distribution compared to no vehicle group (Fig. 7f). Taken together, these results suggest that recurrent muscle injury during the remodeling phase could be alleviated when very low concentration of DMSO was intramuscular administered.

\section{Discussion}

In the current study, the impact of intramuscular administration of lipid-soluble and water-soluble vehicles on skeletal muscle regenerative capacity was investigated. The major findings are (1) the phase of skeletal muscle regeneration (regenerative vs. remodeling) is a crucial factor for determination of the impact of intramuscular administration of vehicle compounds into regenerating muscle; (2) intramuscular administration of high concentrations of DMSO and PG into regenerating muscle leads to recurrent muscle injury that was comparable to $2 \%$ trypsin administration. These deleterious effects were pronounced during the remodeling phase and associated with infiltration of $\mathrm{F} 4 / 80^{+}$macrophages, skeletal muscle stem cell re-activation, nascent regenerating muscle fiber formation, excess fibrous formation, and decreased regenerating muscle fiber CSA; (3) none of the tested vehicles enhanced the regenerative capacity at any phase of skeletal muscle regeneration compared to IGF-1 administration.

Repairing damaged muscle fibers is a spontaneous event after injury that requires skeletal muscle stem cells [1-4] and contributing factors including angiogenesis [7] and extracellular matrix remodeling [8]. These regenerative components act together to ensure complete skeletal muscle regeneration and proper functional recovery. In the present study, there were no significant differences in regenerative muscle fiber formation, satellite cell differentiation, angiogenesis, and fibrous formation in almost tested vehicles when applied at any phase of skeletal muscle regeneration. However, sign of recurrent muscle injury was evident during skeletal muscle regeneration at both regenerative and remodeling phases after 40\% DMSO and 40\% PG administrations and similar in nature with $2 \%$ trypsin administration that served as a degenerative control [24]. In contrast, this deterioration was not observed with $0.9 \% \mathrm{NaCl}$ and distilled water which were the solvents used for preparation of $40 \%$ DMSO and $40 \%$ PG in this study. To support this notion, the myotoxicity of DMSO at high concentration or pure compound was previously tested in isolated EDL muscle of rats using in vitro assays to develop an in situ forming drug delivery system using biodegradable polymers in which the result showing substantial creatine kinase release after administration [26]. Additionally, intramuscular administration of DMSO compound that served as a vehicle induced significant skeletal muscle fiber damage with advanced sarcoma development in treated mice has been reported [27]. Whilst injectable drug formula commonly contains PG as an organic cosolvent, a local muscle damage after intramuscular administration of high concentration or pure compound of PG was demonstrated in rabbit and piglet muscles [28, 29]. This myotoxicity of PG was proposed to be related to the mobilization of $\mathrm{Ca}^{2+}$ homeostasis inside the muscle cell [30]. Nonetheless, all previous studies reported the effects of high concentrations of DMSO and PG on normal skeletal muscle that had a different growth dynamic compared to regenerating muscle [31].

The major finding of this study was that the phase of skeletal muscle regeneration is crucial to determine the effect of the vehicle on modulating skeletal muscle regenerative capacity. Since the impact of recurrent muscle injury after $40 \%$ DMSO and $40 \%$ PG administrations 
Fig. 5 Impact of intramuscular administration of $40 \%$ DMSO, $40 \%$ PG, IGF- 1 , and $2 \%$ trypsin on skeletal muscle regenerative capacity during the remodeling phase. a Representative images of skeletal muscle regeneration regulatory proteins (EbMHC and myogenin) and vimentin protein expression compared to no vehicle. Images were captured at $\times 200$ magnification, scale bars $=50 \mu \mathrm{m}$. b Representative images of EbMHC, myogenin, and vimentin protein expression in the defective areas at $\times 400$ magnification (serial sections are denoted by asterisks), scale bars $=50 \mu \mathrm{m}$. Arrows and arrowheads indicate the localization of nascent regenerating muscle fibers with robust EbMHC and myogenin protein expression, respectively. Laminin was applied to delineate regenerating muscle fiber structure and nuclei were visualized with DAPI

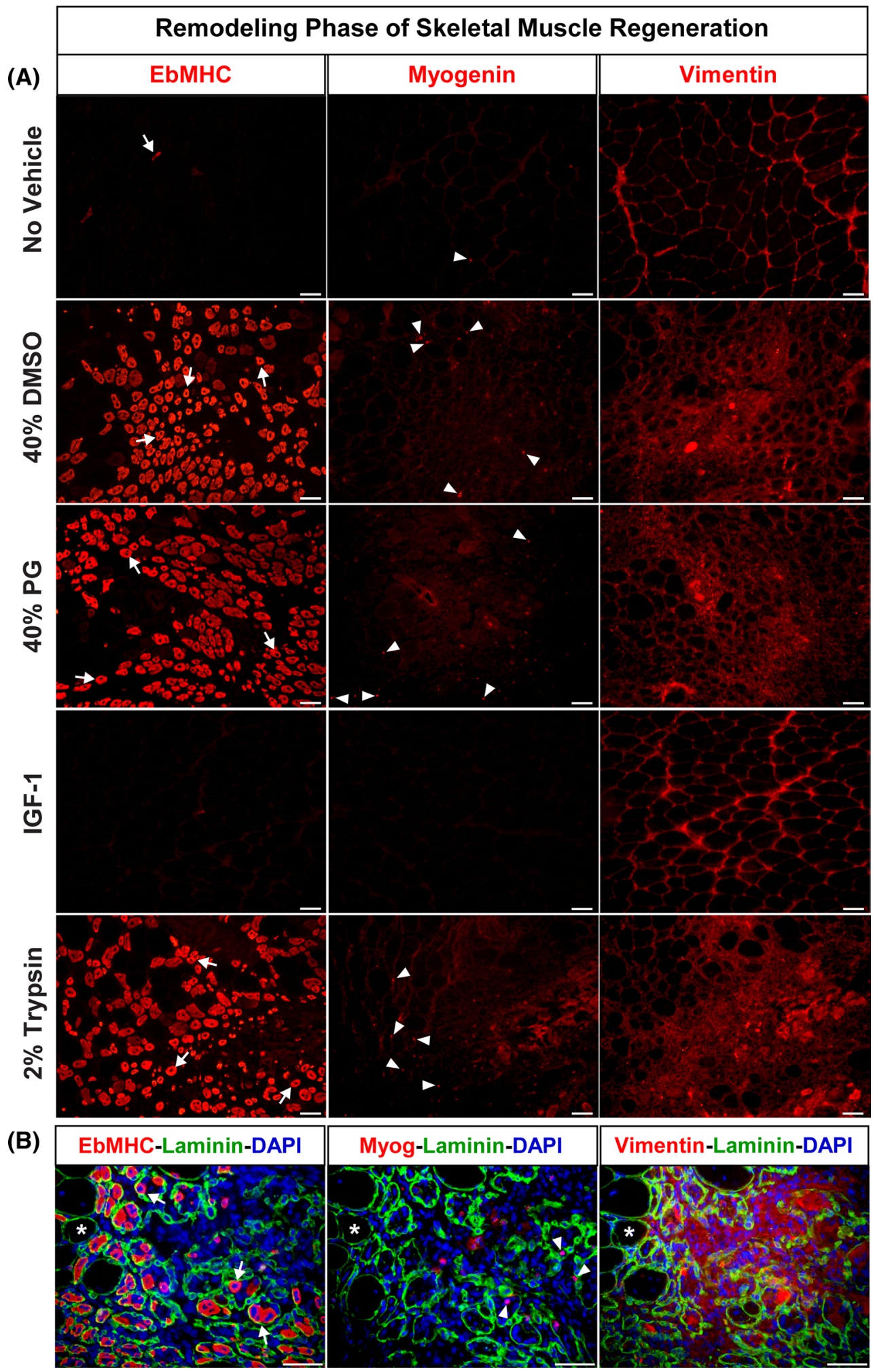

were increased during remodeling compared to regenerative phase of skeletal muscle regeneration, and an exacerbated effect was evident when multiple administrations were applied. According to these findings, the rapid differentiation of satellite cells during the regenerative phase [4] may compensate for the loss of muscle fibers due to the recurrent muscle injury at an early phase of repair. Nevertheless, destruction of regenerating muscle fibers 
Fig. 6 Recurrent muscle injury after $40 \%$ DMSO, 40\% PG, and $2 \%$ trypsin administrations during the remodeling phase of skeletal muscle regeneration. a Representative images of the association of $\mathrm{F} 4 / 80^{+}$ macrophage infiltration (arrowheads) and nascent regenerating muscle fiber formation (EbMHC-stained fibers; arrows) (serial sections are denoted by asterisks). b Representative images of $\mathrm{F} 4 / 80^{+}$macrophage infiltration (arrowheads) after multiple intramuscular administrations of $40 \%$ DMSO, $40 \%$ PG, and $2 \%$ trypsin compared to no vehicle. $\mathbf{a}, \mathbf{b}$ Laminin or dystrophin staining was applied to localize regenerating muscle fiber structure and counterstained with DAPI to visualize nuclei, scale bars $=50 \mu \mathrm{m}$. c Quantitative analysis of $\mathrm{F} 4 / 80^{+}$ infiltrated cells, $* * * p<0.001$ compared to no vehicle $(n=5$ mice/group). d Histogram analysis of regenerating muscle fiber CSA between no vehicle vs. $40 \%$ DMSO, no vehicle vs. $40 \%$ PG, and no vehicle vs. $2 \%$ trypsin administered groups. The average number of measured regenerating muscle fiber CSA of no vehicle, $40 \%$ DMSO, $40 \%$ PG, and $2 \%$ trypsin administered groups were 646 , 865,850 , and 763 fibers/mice, respectively ( $n=5 \mathrm{mice} /$ group)

\section{Remodeling Phase of Skeletal Muscle Regeneration}

(A)

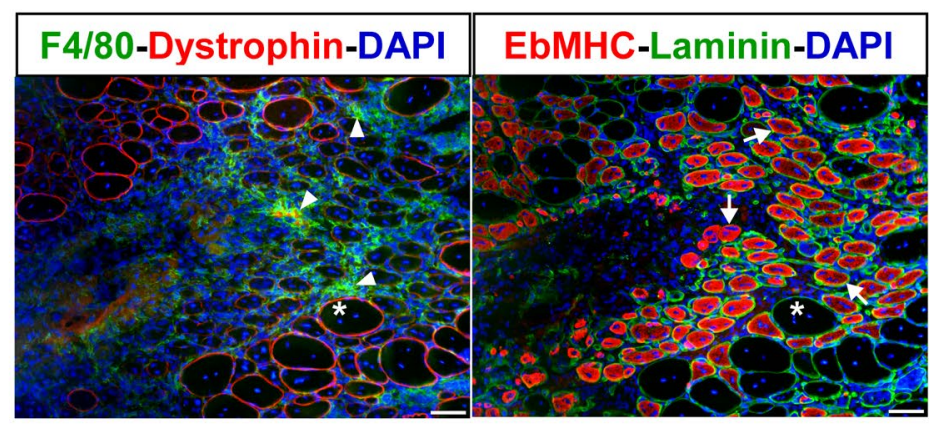

(B)

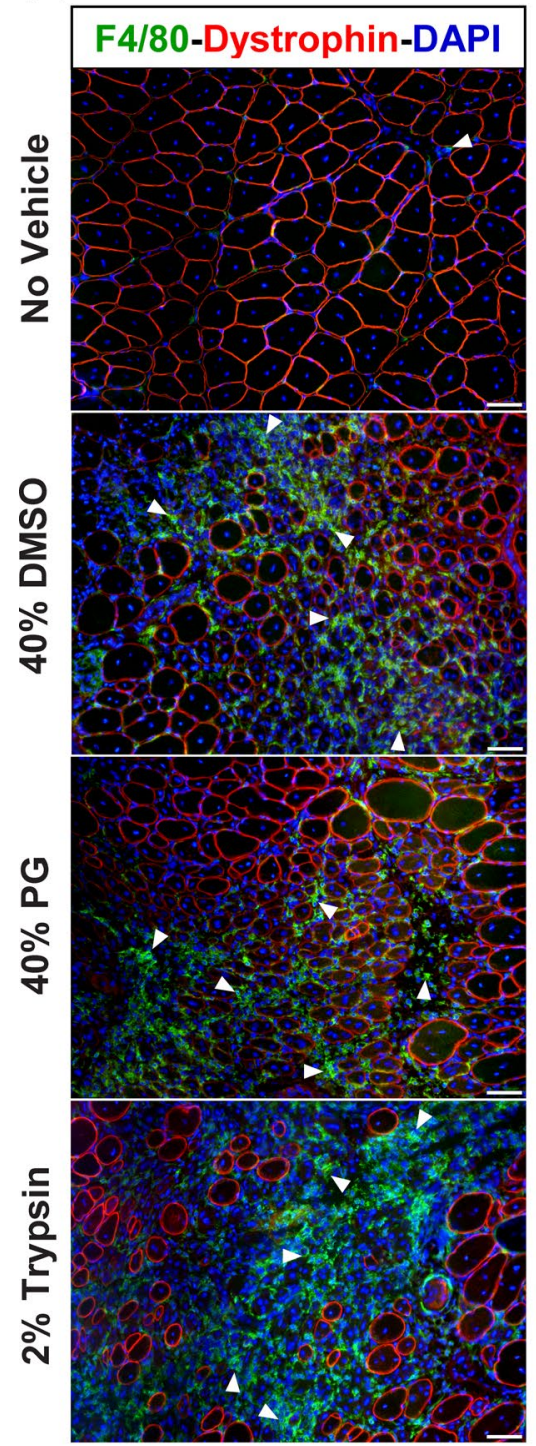

(C)

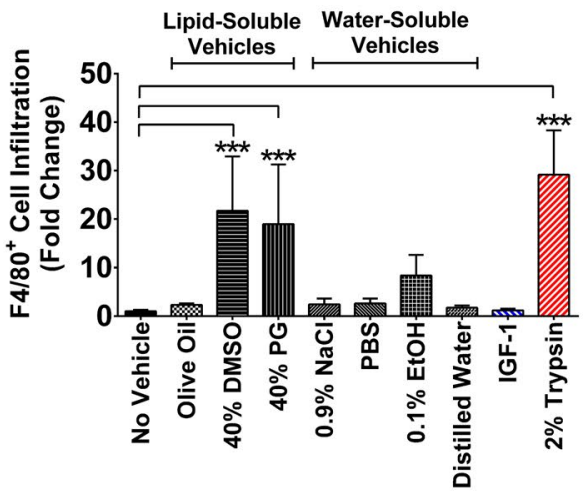

(D)
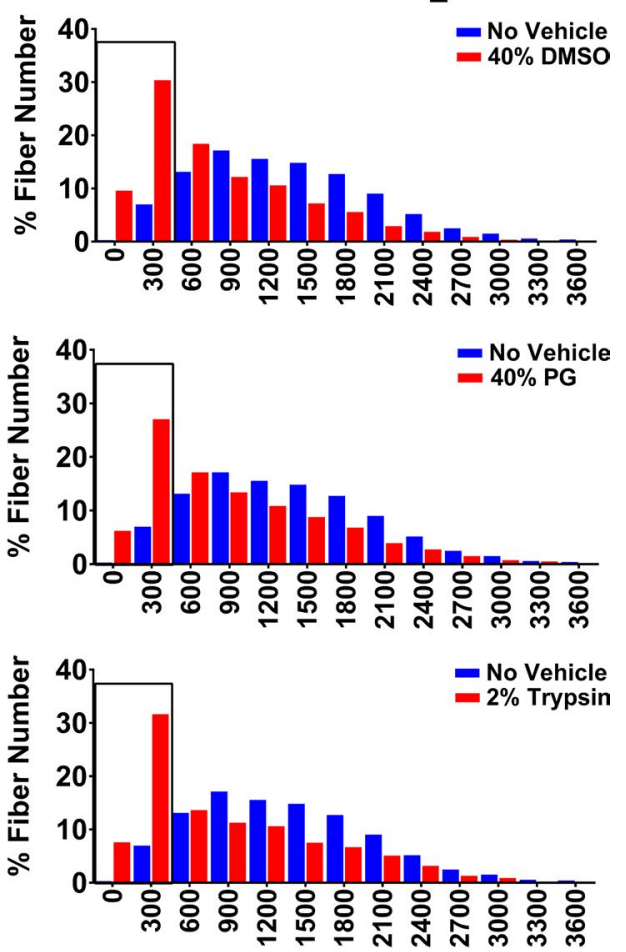

Fiber Cross-Sectional Area $\left(\mu \mathrm{m}^{2}\right)$ 
Fig. 7 Impact of repeated intramuscular administration of 0.1 , 1 , and $5 \%$ DMSO on skeletal muscle regenerative capacity during the remodeling phase. a-c Quantitative analysis of EbMHC, myogenin, and vimentin protein expression, $\mathbf{d ~ F} 4 / 80^{+}$ infiltrated cells, and $\mathbf{e}$ regenerating muscle fiber CSA of no vehicle, $0.1,1$, and $5 \%$ DMSO administered groups. ${ }^{*} p<0.05$ $* * p<0.01$, and $* * * p<0.001$ compared to no vehicle $(n=5$ mice/group). f Histogram analysis of regenerating muscle fiber CSA between no vehicle vs. 5\% DMSO administered group. The average number of measured regenerating muscle fiber CSA of no vehicle and 5\% DMSO administered groups were 646 and 945 fibers/mice, respectively ( $n=5$ mice/group)

\section{Remodeling Phase of Skeletal Muscle Regeneration}

(A)

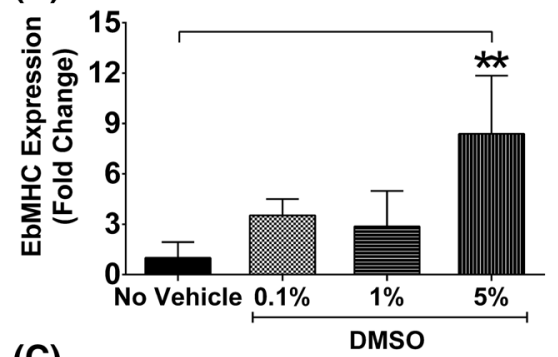

(C)

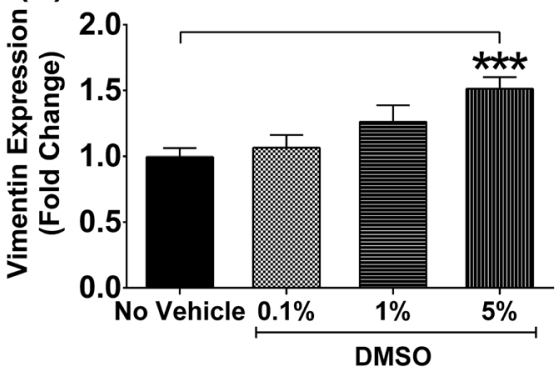

(E)

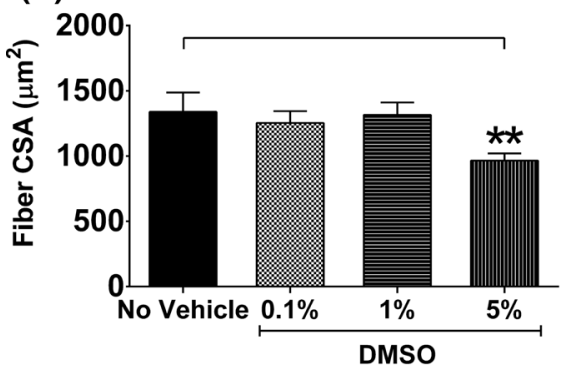

(B)

(D)

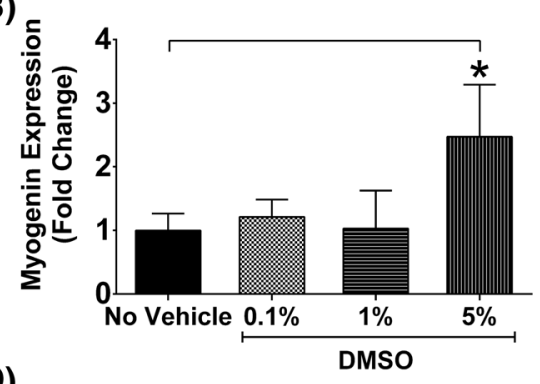

(F)

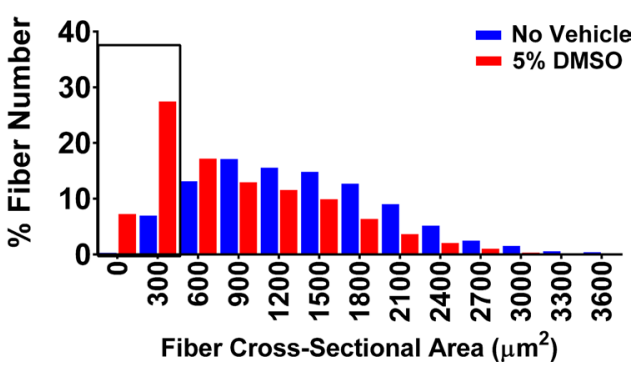

from massive infiltration of $\mathrm{F} 4 / 80^{+}$macrophages was unable to be fully mitigated during the remodeling phase because of reduced activated/differentiated satellite cells at this period [32]. Additionally, a pronounced increase in fibrous formation after intramuscular administrations of $40 \%$ DMSO and 40\% PG at the remodeling phase suggest insufficient of satellite cell activity to repair the damaged muscle fibers. The imbalance between the satellite cell and fibroblast populations during skeletal muscle regeneration could impair proper extracellular matrix remodeling and lead to increased muscular fibrosis [33]. Nevertheless, the deleterious effects of high concentration of DMSO on regenerating muscle fibers during remodeling phase of skeletal muscle regeneration could be alleviated using very low concentration of DMSO (0.1\%). Since recurrent muscle injury was still presented when 5\% DMSO was intramuscular administered into regenerating muscle as demonstrated in the current study.

Besides degenerative effects of high concentration of DMSO and PG administrations, repeated intramuscular administration of $0.1 \% \mathrm{EtOH}$ during the remodeling phase induced a trivial degenerative area. Indeed, this concentration of ethanol is routinely used in skeletal muscle cell culture experiments in vitro without reported myotoxicity [34-36]. These results suggest a dissimilar effect between in vitro and in vivo studies, however, the deleterious effect of $0.1 \% \mathrm{EtOH}$ on skeletal muscle regenerative capacity was minor compared to high concentration of DMSO and PG 
administrations. Furthermore, excess oil accumulation in the subcutaneous (extramuscular space) after multiple administrations of olive oil was observed at the remodeling phase. Extramuscular accumulation of oil could retard regenerating muscle growth as the tendency of decreased muscle wet weight to body weight ratio was evident without the presence of degenerative muscle fibers. Therefore, long-term intramuscular administration of olive oil in the regenerating muscle during remodeling phase should be used with caution.

On the other hand, none of the tested vehicles increased skeletal muscle regenerative capacity at any phase of skeletal muscle regeneration compared to IGF-1. The beneficial effect of IGF-1 on regenerative capacity has been reported elsewhere $[15,17,22]$. In the present study, intramuscular administration of IGF-1 enhanced skeletal muscle regenerative capacity by lowering the levels of EbMHC protein expression during the regenerative phase, suggesting its capability to enhance the maturation of regenerating muscle fibers. However, no significant difference of EbMHC protein expression was detected when IGF-1 was administered at the remodeling phase. The absent effect of IGF-1 at the late stage of regeneration could relate to the point that remodeling phase of skeletal muscle regeneration contains almost $99 \%$ of matured regenerating muscle fibers, therefore, the explicit effect of IGF-1 on enhancement of regenerating muscle fiber maturation is disappear. These results suggest that the beneficial effect of IGF-1 was restricted to the regenerative phase rather than remodeling phase of skeletal muscle regeneration.

Altogether, the phase of skeletal muscle regeneration is a crucial factor for determination of impact of intramuscular administration of vehicles into regenerating muscle. Although none of the tested vehicles in this study had an effect to enhance skeletal muscle regenerative capacity, administration of vehicle compound containing high concentration of DMSO or PG could impair regenerative capacity and potentially affect the validation testing of the investigational substance. Moreover, the findings of this study revealed an important caveat for immunohistochemical-based analysis of skeletal muscle regenerative capacity. The techniques that rely solely on analysis of whole muscle homogenates (i.e., Western blotting, realtime PCR, microarray, etc.) may not be sensitive enough to detect unexpected muscle damage induced by intramuscular administration of an inappropriate vehicle, which could alter typical expressions of the regulatory genes/proteins during skeletal muscle regeneration study.

Acknowledgements The authors gratefully acknowledge the Olympus Bioimaging Center (Faculty of Science, Mahidol University) for technical support on image analysis and Department of Anatomy (Faculty of Science, Mahidol University) for supporting the cryostat for muscle sample sectioning. Proofreading of the manuscript by Assistant Professor Dr. Christopher Fry is appreciated.

\section{Compliance with ethical standards}

Funding This research project was funded by Mahidol University (Talent Management Grant to RS).

Ethical approval All applicable international, national, and/or institutional guidelines for the care and use of animals were followed.

Conflict of interest The authors have no conflicts of interest to declare.

\section{References}

1. Hawke TJ, Garry DJ (2001) Myogenic satellite cells: physiology to molecular biology. J Appl Physiol 91:534-551

2. Relaix F, Zammit PS (2012) Satellite cells are essential for skeletal muscle regeneration: the cell on the edge returns centre stage. Development 139:2845-2856

3. Dumont NA, Bentzinger CF, Sincennes MC, Rudnicki MA (2015) Satellite cells and skeletal muscle regeneration. Compr Physiol 5:1027-1059

4. Shi X, Garry DJ (2006) Muscle stem cells in development, regeneration, and disease. Genes Dev 20:1692-1708

5. Wagner KR, Liu X, Chang X, Allen RE (2005) Muscle regeneration in the prolonged absence of myostatin. Proc Natl Acad Sci USA 102:2519-2524

6. Ciciliot S, Schiaffino S (2010) Regeneration of mammalian skeletal muscle. Basic mechanisms and clinical implications. Curr Pharm Des 16:906-914

7. Arsic N, Zacchigna S, Zentilin L, Ramirez-Correa G, Pattarini L, Salvi A, Sinagra G, Giacca M (2004) Vascular endothelial growth factor stimulates skeletal muscle regeneration in vivo. Mol Ther 10:844-854

8. Goetsch SC, Hawke TJ, Gallardo TD, Richardson JA, Garry DJ (2003) Transcriptional profiling and regulation of the extracellular matrix during muscle regeneration. Physiol Genom $14: 261-271$

9. Grounds MD, Torrisi J (2004) Anti-TNF $\alpha$ (Remicade) therapy protects dystrophic skeletal muscle from necrosis. FASEB J $18: 676-682$

10. Mackey AL, Mikkelsen UR, Magnusson SP, Kjaer M (2012) Rehabilitation of muscle after injury - the role of anti-inflammatory drugs. Scand J Med Sci Sports 22:e8-e14

11. Kobayashi M, Ota S, Terada S, Kawakami Y, Otsuka T, Fu FH, Huard J (2016) The combined use of losartan and musclederived stem cells significantly improves the functional recovery of muscle in a young mouse model of contusion injuries. Am J Sports Med 44:3252-3261

12. Thaloor D, Miller KJ, Gephart J, Mitchell PO, Pavlath GK (1999) Systemic administration of the NF- $\kappa B$ inhibitor curcumin stimulates muscle regeneration after traumatic injury. Am J Physiol 277:C320-C329

13. Toth N, Szabo A, Kacsala P, Heger J, Zador E (2008) 20-Hydroxyecdysone increases fiber size in a muscle-specific fashion in rat. Phytomedicine 15:691-698

14. Miller KJ, Thaloor D, Matteson S, Pavlath GK (2000) Hepatocyte growth factor affects satellite cell activation and differentiation in regenerating skeletal muscle. Am J Physiol Cell Physiol 278:C174-C181 
15. Schertzer JD, Lynch GS (2006) Comparative evaluation of IGF-I gene transfer and IGF-I protein administration for enhancing skeletal muscle regeneration after injury. Gene Ther 13:1657-1664

16. Barnard W, Bower J, Brown MA, Murphy M, Austin L (1994) Leukemia inhibitory factor (LIF) infusion stimulates skeletal muscle regeneration after injury: injured muscle expresses LIF mRNA. J Neurol Sci 123:108-113

17. Takahashi T, Ishida K, Itoh K, Konishi Y, Yagyu KI, Tominaga A, Miyazaki JI, Yamamoto H (2003) IGF-I gene transfer by electroporation promotes regeneration in a muscle injury model. Gene Ther 10:612-620

18. Nozaki M, Li Y, Zhu J, Ambrosio F, Uehara K, Fu FH, Huard J (2008) Improved muscle healing after contusion injury by the inhibitory effect of suramin on myostatin, a negative regulator of muscle growth. Am J Sports Med 36:2354-2362

19. Nakamura Y, Miyaki S, Ishitobi H, Matsuyama S, Nakasa T, Kamei N, Akimoto T, Higashi Y, Ochi M (2015) Mesenchymalstem-cell-derived exosomes accelerate skeletal muscle regeneration. FEBS Lett 589:1257-1265

20. Zhu J, Li Y, Lu A, Gharaibeh B, Ma J, Kobayashi T, Quintero AJ, Huard J (2011) Follistatin improves skeletal muscle healing after injury and disease through an interaction with muscle regeneration, angiogenesis, and fibrosis. Am J Pathol 179:915-930

21. Turner PV, Brabb T, Pekow C, Vasbinder MA (2011) Administration of substances to laboratory animals: routes of administration and factors to consider. J Am Assoc Lab Anim Sci 50:600-613

22. Pelosi L, Giacinti C, Nardis C, Borsellino G, Rizzuto E, Nicoletti C, Wannenes F, Battistini L, Rosenthal N, Molinaro M, Musaro A (2007) Local expression of IGF-1 accelerates muscle regeneration by rapidly modulating inflammatory cytokines and chemokines. FASEB J 21:1393-1402

23. Sato K, Li Y, Foster W, Fukushima K, Badlani N, Adachi N, Usas A, Fu FH, Huard J (2003) Improvement of muscle healing through enhancement of muscle regeneration and prevention of fibrosis. Muscle Nerve 28:365-372

24. Caldwell CJ, Mattey DL, Weller RO (1990) Role of the basement membrane in the regeneration of skeletal muscle. Neuropathol Appl Neurobiol 16:225-238

25. Srikuea R, Hirunsai M (2016) Effects of intramuscular administration of $1 \alpha, 25(\mathrm{OH})_{2} \mathrm{D}_{3}$ during skeletal muscle regeneration on regenerative capacity, muscular fibrosis, and angiogenesis. J Appl Physiol 120:1381-1393
26. Kranz H, Brazeau GA, Napaporn J, Martin RL, Millard W, Bodmeier R (2001) Myotoxicity studies of injectable biodegradable in situ forming drug delivery systems. Int J Pharm 212:11-18

27. Van Mater D, Ano L, Blum JM, Webster MT, Huang W, Williams N, Ma Y, Cardona DM, Fan CM, Kirsch DG (2015) Acute tissue injury activates satellite cells and promotes sarcoma formation via the HGF/c-MET signaling pathway. Cancer Res 75:605-614

28. Ferre PJ, Liaubet L, Concordet D, SanCristobal M, Uro-Coste E, Tosser-Klopp G, Bonnet A, Toutain PL, Hatey F, Lefebvre HP (2007) Longitudinal analysis of gene expression in porcine skeletal muscle after post-injection local injury. Pharm Res 24:1480-1489

29. Brazeau GA, Fung HL (1989) Use of an in vitro model for the assessment of muscle damage from intramuscular injections: in vitro-in vivo correlation and predictability with mixed solvent systems. Pharm Res 6:766-771

30. Brazeau GA, Fung HL (1990) Mechanisms of creatine kinase release from isolated rat skeletal muscles damaged by propylene glycol and ethanol. J Pharm Sci 79:393-397

31. Charge SB, Rudnicki MA (2004) Cellular and molecular regulation of muscle regeneration. Physiol Rev 84:209-238

32. Tidball JG, Villalta SA (2010) Regulatory interactions between muscle and the immune system during muscle regeneration. Am J Physiol Regul Integr Comp Physiol 298:R1173-R1187

33. Murphy MM, Lawson JA, Mathew SJ, Hutcheson DA, Kardon G (2011) Satellite cells, connective tissue fibroblasts and their interactions are crucial for muscle regeneration. Development 138:3625-3637

34. Markworth JF, Cameron-Smith D (2013) Arachidonic acid supplementation enhances in vitro skeletal muscle cell growth via a COX-2-dependent pathway. Am J Physiol Cell Physiol 304:C56-C67

35. Menconi M, Gonnella P, Petkova V, Lecker S, Hasselgren PO (2008) Dexamethasone and corticosterone induce similar, but not identical, muscle wasting responses in cultured L6 and C2C12 myotubes. J Cell Biochem 105:353-364

36. Srikuea R, Zhang X, Park-Sarge OK, Esser KA (2012) VDR and CYP27B1 are expressed in C2C12 cells and regenerating skeletal muscle: potential role in suppression of myoblast proliferation. Am J Physiol Cell Physiol 303:C396-C405 